\title{
Intervención cognitivo-conductual para la mejora del autoconcepto en el caso de una adolescente víctima de maltrato emocional
}

\section{Cognitive-behavioral intervention to enhance self-concept in a case of an adolescent victim of emotional maltreatment}

\author{
Estibaliz Ramos-Díaz ${ }^{(1)}$, Inge Axpe ${ }^{(1)}$, Oihane Fernández-Lasarte ${ }^{(1)}$ y Vanessa Jiménez-Jiménez ${ }^{(2)}$ \\ (1) Universidad del País Vasco/Esukal Herriko Unibertsitatea (UPV-EHU), España \\ (2) Área del Menor y la Familia del Instituto Foral de Bienestar Social de Álava, España
}

\begin{abstract}
Resumen: Los importantes cambios durante la adolescencia en la autopercepción y la decisiva influencia de la imagen propia sobre el bienestar psicológico de los adolescentes determinan el gran interés que despierta el estudio del autoconcepto durante este periodo evolutivo. En el presente estudio se evalúa una intervención cognitivo-conductual dirigida a mejorar el autoconcepto de una adolescente de 16 años que ha sufrido maltrato emocional. Con un diseño de caso único, se aplicó una adaptación del programa de intervención de Mora y Raich (2014) y el programa de intervención "Mírate bien" de Axpe y Revuelta (2012). De la batería de instrumentos utilizada, se administraron dos instrumentos de evaluación antes y después de la intervención: Listado de Síntomas SCL-90-R (Derogatis, Rickels y Rock, 1976) y Cuestionario Autoconcepto Forma-A (AFA) de García y Musitu (2014). Los resultados tras 15 sesiones semanales de intervención muestran una mejora en las puntuaciones del autoconcepto en las dimensiones familiar, emocional y física, así como una disminución de los síntomas psicopatológicos.

Palabras clave: autoconcepto, intervención cognitivo-conductual, diseño de caso único, adolescencia, maltrato emocional.

Abstract: The significant changes during adolescence in self-perception and the decisive influence of the adolescent's own image on psychological well-being determine the great interest in the study of self-concept. The current study evaluates the effectiveness of a cognitive-behavioral intervention to improve the self-concept of a sixteen-year-old adolescent victim of emotional maltreatment. With a single-case design, an adaptation of the intervention program of Mora and Raich (2014) and the "Look at yourself" intervention program of Axpe
\end{abstract}

La correspondencia sobre este artículo debe enviarse a la primera autora al e-mail: estibaliz.ramos@ehu.eus

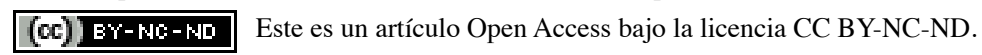


and Revuelta (2012) were applied. From the full instrument battery used, two instruments were administered: Symptom Checklist SCL-90-R (Derogatis, Rickels, \& Rock, 1976) and Self-Concept Form-A Questionnaire (García \& Musitu, 2014). After 15 sessions of intervention, results revealed an improvement in self-concept scores in the family, emotional and physical dimensions, as well as a decrease in psychopathological symptoms.

Keywords: self-concept, cognitive-behavioral intervention, single-case design, adolescence, emotional maltreatment.

Existe una elevada cantidad de recursos personales que los adolescentes deben desarrollar para lograr su bienestar psicológico (Lippman et al., 2014) o el afrontamiento adaptativo de la adversidad (Liu, Wang y Lü, 2013; Wright, Masten y Narayan, 2013). Entre tales activos para el desarrollo adaptativo, la percepción positiva de uno mismo figura como determinante para el ajuste tanto psicológico como social en la adolescencia (Fuentes, García, Gracia y Lila, 2011; Garaigordobil, Pérez y Mozaz, 2008; Inglés, Martínez-Monteagudo, García-Fernández, Valle y Castejón, 2015; Rodríguez-Fernández et al., 2016). El autoconcepto ha sido definido como el conjunto de percepciones (físicas, intelectuales, sociales, académicas, emocionales, etc.) que conforman la imagen que una persona mantiene sobre sí misma a partir de factores cognitivos y de la interacción social (Shavenson, Hubner y Stanton, 1976). Actualmente, este constructo cognitivo-motivacional se entiende como una realidad de naturaleza multidimensional y jerárquica, existiendo una dimensión más general y estable que se sitúa sobre otras más específicas en niveles intermedios e inferiores, como el autoconcepto físico, emocional, familiar, académico o social (Esnaola, Rodríguez-Fernández, y Goñi, 2011; Mérida, Serrano, y Tabernero, 2015; Shavelson, et al., 1976). Durante la adolescencia, el autoconcepto sufre relevantes transformaciones debido al progresivo desarrollo de las habilidades cognitivas, produciéndose una mayor diferenciación del mismo al enfrentarse los adolescentes a nuevas tareas evolutivas, así como a una mayor vulnerabilidad para distorsionar su propia imagen (Inglés, Martínez-González, García-Fernández, Torregrosa, y Ruiz-Esteban, 2012).

Se ha confirmado que un buen autoconcepto resulta esencial en el desarrollo mental saludable durante la adolescencia (Church et al., 2014; Fuentes, Medina, Van Barneveld, y Escobar, 2012; Mruk, 2006). Tener una alta autoestima o autopercepción evaluativa positiva puede ser una cualidad protectora de resultados negativos asociados con la exposición a los riesgos de la adolescencia (Fergus y Zimmerman, 2005). Además, durante este periodo del ciclo vital las autopercepciones positivas están relacionadas con diversos indicadores de adaptación, apoyando la idea de que el mayor autoconcepto se corresponde con el mejor ajuste psicológico, una buena competencia personal y menos problemas comportamentales (Fuentes et al., 2011). Por el contrario, tener una autopercepción negativa puede suponer un riesgo para el bienestar del adolescente; también se han corroborado en adolescentes con autoconcepto positivo relaciones inversas con síntomas de desajuste psicológico (Garaigordobil et al., 2008). Por ello, durante los últimos años el estudio sobre el autoconcepto y la autoestima ha ido ganando relevancia al identificarse como un factor protector directamente implicado con el bienestar general que influye positivamente en las conductas ajustadas y adaptativas en la adolescencia (Garaigordobil et al., 2008; O’Mara, Marsh, Craven, y Debus, 2006; Rodríguez-Fernández et al., 2016).

Todo esto permite comprender el enorme interés por tratar de ayudar a los adolescentes a mejorar u obtener una visión más ajustada de su self mediante su implicación en intervenciones psicológicas eficaces. En este sentido, la Asociación Americana de Psicología (APA) subraya la importancia de una perspectiva proactiva y preventiva en relación con la salud psicológica (Hage et al., 2007). Las pautas planteadas implican un doble proceso: por un lado, la promoción de acciones necesarias para disminuir los problemas y, por otro lado, el fortalecimiento de los factores que conducen al desarrollo positivo (Kenny y Hage, 2009). Dentro de este marco, el trabajo de las creencias y pensamientos de la persona acerca de sí misma es fundamental para la mejora del autoconcepto (Axpe, Infante, y Goñi, 2016). Efectivamente, se ha verificado a nivel empírico que los programas de aprendizaje socioemocional con 
adolescentes promueven la mejora del autoconcepto general y la autoestima (Coelho, Sousa, y Figueira, 2014; Morton y Montgomery, 2013). También se ha verificado la eficacia de un programa de intervención sobre el autoconcepto físico implementado desde una perspectiva cognitiva que permite que la persona aplique y generalice las estrategias cognitivas aprendidas a otros ámbitos de la propia percepción, de manera que el autoconcepto general resulta igualmente beneficiado (Axpe, Gil, e Iriarte, 2012; Axpe, Infante y Goñi, 2016).

Por otro lado, varias investigaciones estudian las consecuencias negativas que puede generar el maltrato emocional en el funcionamiento psicológico durante la infancia y la adolescencia. Los malos tratos en la infancia constituyen un importante factor de riesgo psicopatológico y se relacionan con conductas disfuncionales en la adolescencia (Lázaro y López, 2010; Shaffer, Huston y Egeland, 2008). Precisamente, es constatable que determinados procedimientos de intervención destinados a potenciar habilidades emocionales de afrontamiento ante la adversidad han mostrado su eficacia para producir cambios significativos en adolescentes (Prince-Embury y Saklofske, 2014). No obstante, no se encuentran estudios que analicen la relación entre el maltrato infantil y el papel de la intervención sobre el autoconcepto con el objetivo de disminuir tales consecuencias psicopatológicas. La investigación sobre el efecto de las intervenciones psicológicas que promueven el desarrollo del autoconcepto y la autoestima con adolescentes expuestos a situaciones de maltrato es limitada en la actualidad. Dada la centralidad psicológica del autoconcepto, resulta relevante ampliar las investigaciones que favorezcan el conocimiento de la eficacia de este tipo de intervenciones con adolescentes que cuentan con antecedentes de maltrato.

El presente estudio tiene como objetivo evaluar los efectos de una intervención psicológica para mejorar el autoconcepto de una adolescente víctima de maltrato grave. Este trabajo ha utilizado como base el programa de tratamiento de Mora y Raich (2014) y el programa de intervención "Mírate bien. Claves para mejorar la imagen propia y el bienestar psicológico" de Axpe y Revuelta (2012). De forma específica, se trata de entrenar a la paciente en una serie de habilidades cognitivas y sociales que puedan mejorar la imagen de sí misma y favorecer su bienestar general.

\section{Presentación del caso}

\section{Identificación del paciente}

Paciente adolescente de 16 años de edad escolarizada en cuarto curso de Educación Secundaria Obligatoria. Vive con una familia de acogida y su hermano menor tras haber experimentado maltrato infantil. Según la entrevista con los padres acogedores, la adolescente presenta un buen rendimiento académico y una adecuada relación con los iguales. Se trata de una menor que presenta ajuste en el contexto escolar y que se encuentra en proceso de adaptación a la situación de acogimiento familiar. Entre sus aficiones destacan la lectura y dedicar tiempo libre a estar con su grupo de amistades. El nivel sociocultural de la familia es medio-bajo y existe un vínculo afectivo positivo entre todos los miembros de la familia.

\section{Motivo de consulta}

La demanda de apoyo psicológico por parte de la familia acogedora y la psicóloga coordinadora del caso en el Área del Menor y la Familia se centra fundamentalmente en la consolidación el proceso de formación de la identidad personal de la menor y la mejora de su autoconcepto. Asimismo, se considera necesaria la creación de un espacio de desahogo personal debido al retraimiento que presenta la joven, caracterizado fundamentalmente por la falta de accesibilidad a iniciar intercambios emocionales dentro del contexto familiar. La adolescente, por su parte, solicita ayuda específica para mejorar la percepción negativa que tiene de sí misma y que influye en su estado de ánimo, presentando síntomas depresivos. 


\section{Historia del problema}

La paciente es una adolescente con antecedentes de maltrato emocional que presenta un perfil resiliente, describiéndose a sí misma como una chica tímida, incomprendida, sensible y empática. Presenta evaluaciones negativas sobre sí misma, fundamentalmente percepciones distorsionadas sobre su apariencia física, inseguridad e insatisfacción por la imagen propia. Muestra tendencia a aceptar valoraciones desfavorables sobre sí misma como correctas.

En su familia acogedora, la menor percibe una adecuada cohesión familiar y un bajo nivel de conflicto, aunque admite que la comunicación no es fluida por su parte y que le cuesta expresar su punto de vista. El entorno familiar intenta reforzar la introspección y la comunicación emocional, pero su inaccesibilidad dificulta que las figuras acogedoras puedan comprender su estado de ánimo. No obstante, describe una buena relación con sus padres acogedores, confía en ellos y destaca que se interesan por ella, se implican con ella y le ayudan.

A nivel social, cuenta con un grupo reducido de amistades íntimas con el que siente segura y confiada. Debido al reciente cambio de centro educativo, la interacción con los compañeros y compañeras de clase se reduce a las horas lectivas. Las autopercepciones negativas que presenta dificultan un adecuado desempeño social y la adecuación interpersonal con posibles nuevas amistades en el nuevo entorno escolar. No participa en ninguna actividad extraescolar de ocio, deportiva o académica.

\section{Evaluación}

Para realizar la evaluación psicológica se recogió información relevante sobre el caso en varias entrevistas concertadas con profesionales del programa de apoyo psicológico de los servicios sociales. También se llevaron a cabo entrevistas clínicas con la madre acogedora y la paciente por separado. A continuación se exponen los instrumentos de evaluación empleados en la exploración psicológica inicial y los resultados obtenidos:

Cuestionario "Big Five" de Personalidad BFQ (adaptación española: Bermúdez, 1995). La prueba identifica un perfil cooperativo, cordial, altruista, amigable, generoso y empático de la paciente. La puntuación implica asimismo una alta capacidad para comprender los problemas y necesidades de los demás y cooperar eficazmente con ellos. Obtiene un índice alto que sugiere la tendencia a definirse como muy reflexiva, escrupulosa, diligente y perseverante. Por último, los resultados sugieren que la paciente mantiene el control de la propia conducta en situaciones difíciles y que presenta niveles bajos de impulsividad, emotividad, impaciencia e irritabilidad.

Listado de Síntomas SCL-90-R (Derogatis et al., 1976). Se ubica en el nivel de personas sin morbilidad psicopatológica, tanto en el índice global como en las dimensiones sintomáticas. Las puntuaciones más elevadas corresponden a las escalas de sensibilidad interpersonal y de depresión. La primera dimensión está relacionada con un estado de incomodidad e inhibición ante el sexo opuesto, la impresión de que los demás no la comprenden y la percepción de una misma como extremadamente sensible o sentirse herida con facilidad. La segunda dimensión recoge signos relacionados con sentimientos de tristeza y llorar fácilmente.

Test Autoevaluativo Multifactorial de Adaptación Infantil TAMAI (Hernández, 1990). En el factor de inadaptación personal obtiene una puntuación moderada. El sistema de indicación crítica señala un nivel moderado del factor de cogniafección, un nivel casi alto de cognipunición y un nivel bajo de autosuficiencia defensiva. La primera dimensión implica una tendencia moderada a estar insatisfecha consigo misma y sensaciones de incomodidad, inseguridad y miedo de enfrentarse a la realidad; la segunda dimensión sugiere niveles altos de sufrimiento personal; $\mathrm{y}$ la tercera dimensión alude a la tendencia a expresar una posible inadaptación a través de sentimientos negativos de tristeza en lugar de reacciones defensivas. En las áreas de inadaptación escolar y social, ninguno de los factores (aversión a la instrucción, indisciplina, autodesajuste social y restricción social) aparecen como constatados.

Escala de Clima Familiar FES (adaptación española: Fernández-Ballesteros y Sierra, 1984). El análisis de los resultados sugiere que percibe un nivel alto de cohesión familiar, y niveles bajos de conflicto y de expresividad en el ámbito familiar. 
Cuestionario Autoconcepto Forma-A (AFA) de García y Musitu (2014). El resultado de la prueba refleja un nivel alto en la dimensión autoconcepto académico y autoconcepto social. Por otra parte, obtiene puntuaciones bajas en el resto de las dimensiones: autoconcepto emocional, autoconcepto familiar y autoconcepto físico.

Asimismo, se solicita a la paciente elaborar un autorregistro de situación-pensamiento-emoción-conducta con el objetivo de poder reconocer el papel que juegan sus pensamientos negativos en lo que siente (sus emociones) y lo que hace (su conducta). Para ello, debía describir la situación o suceso que había motivado la emoción desagradable, los pensamientos automáticos que precedían la emoción negativa y el nivel de creencia de estos pensamientos (valorado de 0 a 10), las emociones negativas que sentía y su intensidad (valorada de 0 a 10), y finalmente qué hacía en esa situación y qué consecuencias tenía ese comportamiento.

Respecto a las conductas llevadas a cabo, se observa evitación. Tanto el grado de creencia de los pensamientos negativos y la intensidad de las emociones fue alto.

\section{Análisis funcional}

\section{Experiencia temprana}

Abandono materno, negligencia, maltrato emocional, comparaciones desfavorables del padre biológico, comentarios laudatorios del padre biológico a hermanos de sexo masculino

\section{Creencias centrales}

"No me gusta mi aspecto físico", "Me gustaría nacer de nuevo y ser distinta de cómo soy", "Me tengo rabia a mí misma" "Me da miedo la gente, me cuesta confiar"

\section{Reglas de vida}

"Soy desconfiada con los demás", "Necesito ser aceptada y querida por los demás, pero debo protegerme para que no me hagan daño", "Si la gente sabe cómo soy realmente me rechazará"

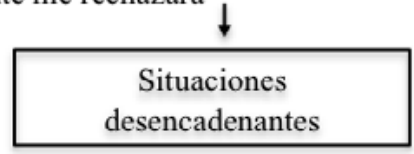

Insatisfacción y confusión con su vida tras los cambios vitales sucedidos percibidos como amenazantes, incertidumbre acerca de su situación de acogimiento familiar, solicitud de la salida de la familia acogedora para ingresar en un centro, hermetismo emocional con la familia acogedora, rechazo de opciones de socialización en el nuevo centro educativo

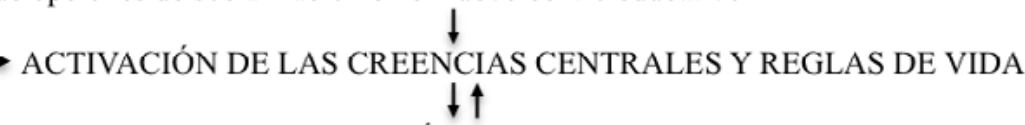

PENSAMIENTOS AUTOMÁTICOS Y DISTORSIONES COGNITIVAS

"No sé lo que quiero"

"Me cuesta mucho comunicarme emocionalmente con mi familia"

“Cómo fiarme de los demás si antes prometieron que nunca me abandonarian y no lo cumplicron?"

"Creo que no gustaré a nadie"

EMOCIONES NEGATIVAS

(síntomas depresivos)

\section{$\downarrow \uparrow$}

\section{COMPORTAMIENTOS DESADAPTADOS}

Problemas interpersonales con los padres acogedores, indefinición personal, disminución del nivel de actividad general o pasividad, encierro en sí misma y aislamiento dentro de la familia, reducción de intereses, inhibición emocional, evitación de situaciones interpersonales que impliquen apertura emocional 


\section{Tratamiento}

El tratamiento cognitivo-conductual dirigido a mejorar el autoconcepto de la paciente se realizó mediante terapia cognitiva-conductual, basado principalmente en técnicas de auto-observación y reestructuración cognitiva, entrenamiento de habilidades sociales y solución de problemas. Se llevaron a cabo asimismo técnicas dirigidas a promover la autoaceptación y la consolidación de puntos fuertes de la autoestima o autoconcepto. Esta intervención psicológica estuvo basada en la propuesta de tratamiento de Mora y Raich (2014).Por último, se aplicó una adaptación del programa de intervención para mejorar la imagen propia y el bienestar psicológico "Mírate bien" de Axpe y Revuelta (2012). Se llevaron a cabo un total de 15 sesiones de tratamiento con una duración aproximada de sesenta minutos cada sesión. Fue aplicada de manera individual en el gabinete de psicología al que se derivó el caso. La periodicidad fue semanal y tuvo una duración de 5 meses aproximadamente.

\section{Objetivos}

- Mejora de la autopercepción y del estado de ánimo a través de la detección y modificación de los pensamientos automáticos autocríticos que son fuente de estados emocionales negativos y comportamientos desadaptados

- Fomentar la autoaceptación a través de la adquisición de un conocimiento más preciso de sus puntos fuertes y puntos débiles

- Identificación y modificación de reglas de vida

- Entrenamiento en habilidades sociales para optimizar las relaciones interpersonales, de comunicación y relacionales

- Mejora de la autoimagen y el bienestar psicológico

\section{Intervención con la paciente}

Las sesiones de intervención cognitivo-conductual se desarrollaron en cinco fases (véase Tabla 1) cuya finalidad fue principalmente mejorar el autoconcepto, disminuir las conductas desadaptadas asociadas a las autopercepciones negativas y favorecer el bienestar psicológico, dotando de habilidades de interacción social.

En la primera fase el objetivo es la intervención sobre los pensamientos automáticos a través de estrategias de codificación y procesamiento de la información como la interpretación y cuestionamiento de las disfunciones cognitivas frecuentes, psicoeducación de la paciente sobre su estructura cognitiva, sus creencias disfuncionales y sus distorsiones cognitivas, corrección de distorsiones cognitivas y mejora de la capacidad para regular el estilo de procesamiento de la información. Asimismo, forma parte de esta fase la enseñanza de habilidades de solución de problemas a través de experimentos conductuales ajustados a la realidad de la paciente.

En la segunda fase, el objetivo es la promoción de la autopercepción positiva intentando fortalecer las valoraciones positivas de sí misma a través de la creación de un diario de actividades en el que se relatan actividades relacionadas con sus éxitos y experiencias positivas, así como la elaboración de una lista de cualidades positivas o logros.

En la tercera fase, el objetivo es la modificación de las cogniciones profundas fuente de comportamientos desadaptativos. Para ello, se identifican y se modifican las reglas de vida de la paciente a través de interrogación socrática, experimentos conductuales, debate didáctico y análisis de la validez o puesta a prueba de las reglas. Además, se analizan los temas más recurrentes en base a los autorregistros de pensamientos automáticos.

La cuarta fase tiene como objetivo la mejora de la calidad de las relaciones interpersonales, de comunicación y relacionales. Basada en el entrenamiento autoinstruccional y de afrontamiento de críticas, se utilizan textos informativos acerca de la asertividad y preguntas finales para comprobar la comprensión de los contenidos trabajados. Asimismo, se implementan técnicas de entrenamiento en habilidades sociales como el modelado, ensayos conductuales basados en experiencias reales, feedback y reforzamiento. También se proponen tareas para casa del tipo exposición a situaciones evitadas. 
Finalmente, la quinta fase tiene como objetivo la mejora de la imagen propia y del bienestar psicológico mediante la adaptación del programa "Mírate bien. Claves para mejorar la imagen propia y el bienestar psicológico" de Axpe y Revuelta (2012).Se trata de un programa en formato impreso que consta de una unidad introductoria, seguida de varios capítulos que abordan el ejercicio físico, los hábitos saludables, las presiones externas y otras variables que afectan a las autopercepciones físicas y la modificación de la propia visión subjetiva.

Una vez finalizada la quinta fase, la evaluación postratamiento se llevó a cabo en una sesión complementaria. Hubo seguimiento del caso por parte de los servicios sociales a través del programa especializado de intervención familiar, informándose de una evolución terapéutica favorable.

Tabla 1. Cronograma de Sesiones de Evaluación y Tratamiento

\begin{tabular}{|c|c|c|c|}
\hline Sesión & Fase & Objetivos & Componentes básicos \\
\hline $1,2,3$ & Evaluación & $\begin{array}{l}\text { - Entrevista abierta y cuestionarios } \\
\text { - Explicar Análisis Funcional, } \\
\text { objetivos y tratamiento }\end{array}$ & - Entrevista abierta \\
\hline $4,5,6$ & $\begin{array}{l}\text { Fase } 1 \\
\text { Intervención sobre } \\
\text { los pensamientos } \\
\text { automáticos }\end{array}$ & $\begin{array}{l}\text { - Identificar los pensamientos } \\
\text { automáticos } \\
\text { - Corrección de los } \\
\text { pensamientos automáticos }\end{array}$ & $\begin{array}{l}\text { - Registro de pensamientos automáticos y su debate } \\
\text { - Corrección de pensamientos automáticos } \\
\text { por interrogación socrática } \\
\text { - Experimento conductual }\end{array}$ \\
\hline $6,7,8$ & $\begin{array}{l}\text { Fase } 2 \\
\text { Autopercepción } \\
\text { positiva }\end{array}$ & · Promover la autoaceptación & $\begin{array}{l}\text { - Identificación de puntos fuertes y } \\
\text { débiles de la autoestima } \\
\text { - Consolidación de los puntos } \\
\text { fuertes de la autoestima } \\
\text { - Diario de actividades } \\
\text { - Lista de méritos }\end{array}$ \\
\hline $\begin{array}{l}9,10, \\
11,12\end{array}$ & $\begin{array}{l}\text { Fase } 3 \\
\text { Intervención sobre } \\
\text { creencias centrales }\end{array}$ & $\begin{array}{l}\text { - Identificar y modificar } \\
\text { reglas de vida }\end{array}$ & $\begin{array}{l}\text { - Interrogación socrática } \\
\text { - Afirmaciones directas } \\
\text { - Análisis de temas comunes emergentes en los } \\
\text { autorregistros de pensamientos automáticos } \\
\text { - Modificación a través de } \\
\text { experimentos conductuales } \\
\text { - Modificación a través de debate didáctico } \\
\text { - Modificación a través de la } \\
\text { interrogación socrática } \\
\text { - Análisis de la validez de las reglas }\end{array}$ \\
\hline $\begin{array}{l}13,14, \\
15\end{array}$ & $\begin{array}{l}\text { Fase } 4 \\
\text { Entrenamiento } \\
\text { en habilidades }\end{array}$ & $\begin{array}{l}\text { - Mejora de la calidad de las } \\
\text { relaciones interpersonales, de } \\
\text { comunicación y relacionales }\end{array}$ & $\begin{array}{l}\text { - Entrenamiento en afrontamiento de críticas } \\
\text { - Entrenamiento autoinstruccional } \\
\text { - Textos informativos y preguntas de comprensión } \\
\text { - Modelado } \\
\text {-Ensayo conductual } \\
\text { - Feedback } \\
\text { - Reforzamiento } \\
\text { - Asignación de tareas para casa: técnica } \\
\text { de exposición, autoinstrucciones }\end{array}$ \\
\hline $\begin{array}{l}15,16 \\
17\end{array}$ & $\begin{array}{l}\text { Fase } 5 \\
\text { Programa } \\
\text { "Mírate bien", }\end{array}$ & $\begin{array}{l}\text { - Mejora de la imagen propia } \\
\text { y del bienestar psicológico } \\
\text { - Promoción de hábitos saludables } \\
\text { implicados en el mantenimiento } \\
\text { de la salud física y mental } \\
\text { - Toma de conciencia de los } \\
\text { influjos socioculturales }\end{array}$ & $\begin{array}{l}\text { - Textos informativos y preguntas de comprensión } \\
\text { - Test de autoconocimiento } \\
\text { - Reestructuración cognitiva } \\
\text { - Motivación para el cambio } \\
\text { - Planificación del cambio (objetivos concretos) }\end{array}$ \\
\hline
\end{tabular}




\section{Resultados tras la intervención}

En base a los resultados obtenidos a través de los instrumentos de evaluación administrados antes y después del tratamiento cognitivo-conductual, se observa una evolución positiva en las dimensiones del autoconcepto de la paciente, así como una disminución de los niveles de sensibilidad interpersonal y depresión. Concretamente, los datos indican que la paciente mantiene una percepción positiva de la calidad del desempeño de su rol como estudiante (autoconcepto académico) tanto en el sentimiento que tiene a partir de sus profesores como en las cualidades específicas valoradas especialmente en el contexto escolar $\left(\mathrm{PT}_{\text {(prestest) }}=19 ; \mathrm{PT}_{\text {(postest) }}=\right.$ 17). Asimismo preserva una percepción positiva en relación a su desempeño en las relaciones sociales (autoconcepto social) cuando se refieren a su red social y a las cualidades importantes en las relaciones interpersonales $\left(\mathrm{PT}_{\text {(prestest) }}=22 ; \mathrm{PT}_{\text {(postest) }}=24\right)$. Respecto al autoconcepto emocional, las puntuaciones obtenidas sugieren un avance positivo de su estado emocional y de sus respuestas a situaciones específicas donde la otra persona implicada es de un rango superior $\left(\mathrm{PT}_{\text {(prestest) }}=19 ; \mathrm{PT}_{\text {(postest) }}=8\right)$. Esto implica que ha disminuido la intensidad de su malestar emocional y que tiene más control de las situaciones y emociones y que responde más adecuadamente a los diferentes momentos de su vida. La paciente manifiesta mejora en el autoconcepto familiar, sobre todo en la implicación, participación e integración en el medio familiar actual ( $\mathrm{PT}_{\text {(prestest) }}=10 ; \mathrm{PT}_{\text {(postest) }}=14$ ), disminuyendo asimismo la percepción de ser criticada en casa y sentir que es una decepción para su familia. Además, se comprueba una mejora en el autoconcepto físico, especialmente en la dimensión de aspecto físico. Esto último implica que se percibe físicamente más agradable en comparación con la percepción negativa pretratamiento $\left(\mathrm{PT}_{\text {(prestest) }}=8 ; \mathrm{PT}_{\text {(postest) }}=16\right.$ ).

Respecto a la evolución de las características psicopatológicas evaluadas con el SCL-90-R, la paciente manifiesta una disminución de los síntomas depresivos $\mathrm{PT}_{(\text {prestest })}=3.54 ; \mathrm{PT}_{\text {(postest) }}=2.61$ ) asociados a la percepción de sí misma, así como una mejora la dimensión de sensibilidad interpersonal $\left(\mathrm{PT}_{\text {(prestest) }}=4.6 ; \mathrm{PT}_{\text {(postest) }}=3.8\right.$ ) relacionada con sentimientos de incomprensión y la percepción de sí misma como extremadamente sensible.

Además de administrar al final de la intervención psicológica la batería de instrumentos destinados a medir el autoconcepto multidimensional y las características psicopatológicas con el objetivo de comparar los niveles prestes y postest, se entrevistó nuevamente a la familia acogedora y se mantuvo una reunión informativa con la responsable del programa especializado de intervención familiar. Dentro del entorno familiar, la paciente se muestra más accesible, aumentando el nivel de comunicación con sus padres acogedores. Tras la intervención, la joven manifiesta una alta capacidad para identificar sus propias cogniciones con los correspondientes correlatos emocionales, físicos y conductuales. Asimismo, es capaz de identificar los pensamientos automáticos negativos y de llevar a cabo una reestructuración cognitiva adaptativa basada en la autoaceptación.

\section{Discusión}

Los resultados de este estudio son consistentes con datos obtenidos en investigación previa que han verificado a nivel empírico el efecto positivo de intervenciones socioemocionales en la promoción de la mejora del autoconcepto general (Coelho et al., 2014; Morton y Montgomery, 2013) y su dimensión física (Axpe et al., 2016; Axpe et al., 2016) durante la adolescencia. Además, se confirma el relevante rol del autoconcepto como un factor estrechamente vinculado al ajuste personal y social en la adolescencia (Rodríguez-Fernández et al., 2016; Ramos-Díaz, Rodríguez-Fernández, Fernández-Zabala, Revuelta y Zuazagoitia, 2016).

\section{Conclusión}

Los objetivos de la intervención psicológica llevada a cabo fueron la mejora del autoconcepto y del bienestar psicológico de una adolescente de 16 años víctima de maltrato infantil. En base a los resultados obtenidos, se cumplieron los objetivos de la intervención. Por un lado, la paciente mejoró las puntuaciones del autoconcepto familiar, autoconcepto emocional y autoconcepto físico. Por otro lado, se confirmó una disminución de 
los síntomas psicopatológicos detectados al inicio del tratamiento. Respecto al curso de la intervención, se observa una adecuada adherencia terapéutica por parte de la paciente. Se subrayan como aspectos cruciales en la evolución positiva de la paciente su participación activa en el proceso terapéutico y la motivación para el cambio manifestada, así como el compromiso y la coordinación entre la psicoterapeuta, los servicios sociales y la familia acogedora.

Por último, el estudio presenta algunas limitaciones que deben ser señaladas. Al tratarse de un diseño de caso único, la generalización de los resultados se ve condicionada, por lo que sería interesante desarrollar nuevas investigaciones que pudieran replicar los efectos en una muestra amplia de población adolescente en situación de desprotección. Sería asimismo conveniente realizar un seguimiento a largo plazo y evaluar la variabilidad temporal de la joven y la estabilidad de los efectos de la intervención cognitivo-conductual a través de replicaciones a nivel intrasujeto para mejorar la validez interna del estudio.

\section{Referencias}

Axpe, I., Gil, I. N. e Iriarte, A. A. (2012). Intervención para la mejora del autoconcepto físico en la adolescencia. Revista Internacional de Ciencias Sociales, 1(2). Recuperado de http://journals .epistemopolis.org/index. $\mathrm{php} / \mathrm{csociales} /$ article/view/1211

Axpe, I., Infante, G. y Goñi, E. (2016). Mejora del autoconcepto físico: eficacia de una intervención cognitiva breve con alumnado universitario de educación primaria. Educación XXI, 19(1), 227-245. https://doi. org/10.5944/educXX1.14476

Axpe, I. y Revuelta, L. (2012). Un programa de intervención cognitiva sobre el autoconcepto físico. Revista Internacional de Educación y Aprendizaje, 1(2), 17-30.

Bermúdez, J. (1995). Cuestionario "Big Five”. Adaptación al castellano del cuestionario BFQ de Caprara, Barbaranelli y Borgogni (1993). Madrid, España: TEA Ediciones.

Church, A. T., Katigbak, M. S., Ibáñez-Reyes, J., de Jesús Vargas-Flores, J., Curtis, G. J., Tanaka-Matsumi, J., ... Simon, J. R. (2014). Relating self-concept consistency to hedonic and eudaimonic well-being in eight cultures. Journal of Cross-Cultural Psychology, 45, 695-712. https://doi.org/10.1177/0022022114527347

Coelho, V., Sousa, V. y Figueira, A. P. (2014). The Impact of a School-Based Social and Emotional Learning Program on the Self-Concept of Middle School Students. Revista de Psicodidáctica, 19, 347-365. https://doi. org/10.1387/RevPsicodidact.10714

Derogatis, L. R., Rickels, K. y Rock, A. F. (1976). The SCL-90 and the MMPI: a step in the validation of a new self-report scale. The British Journal of Psychiatry, 128, 280-289.

Esnaola, I., Rodríguez-Fernández, A. y Goñi, E. (2011). Propiedades psicométricas del Cuestionario de Autoconcepto AFA 5. Anales de Psicología, 27, 109-117.

Fergus, S. y Zimmerman, M. A. (2005). Adolescent resilience: A framework for understanding healthy development in the face of risk. Annual Review of Public Health, 26, 399-419. https://doi.org/10.1146/annurev. publhealth.26.021304.144357

Fernández-Ballesteros, R. y Sierra, B. (1984). Escalas de clima social: familia, trabajo, instituciones penitenciarias, centro escolar. Manual: Investigación y publicaciones psicológicas. Madrid, España: TEA Ediciones. Fuentes, M. C., García, J., Gracia, E. y Lila, M. (2011). Autoconcepto y ajuste psicosocial en la adolescencia. Psicothema, 23, 7-12.

Fuentes, N., Medina, J., Van Barneveld, H. y Escobar, S. (2012). Resiliencia y factores protectores en menores infractores y en situación de calle. Psicología y Salud, 22, 49-62.

Garaigordobil, M., Pérez, J. y Mozaz, M. (2008). Self-concept, self-esteem and psychopathological symptoms. Psicothema, 20(1), 114-123.

García, F. y Musitu, G. (2014). AF-5. Autoconcepto Forma 5. Madrid, España: TEA Ediciones.

Hage, S. M., Romano, J. L., Conyne, R. K., Kenny, M., Matthews, C., Schwartz, J. P. y Waldo, M. (2007). Best practice guidelines on prevention practice, research, training, and social advocacy for psychologists. The Counseling Psychologist, 35, 493-566. https://doi.org/10.1177/0011000006291411 
Hernández, P. (1990). TAMAI. Test Autoevaluativo Multifactorial de Adaptación Infantil. Madrid, España: TEA Ediciones.

Inglés, C. J., Martínez-González, A. E., García-Fernández, J. M., Torregrosa, M. S. y Ruiz-Esteban, C. M. (2012). Prosocial Behavior and Self-Concept of Spanish Students of Compulsory Secondary Education. Revista de Psicodidáctica, 17, 135-156.

Inglés, C., Martínez-Monteagudo, M., García-Fernández, J., Valle, A. y Castejón, J. (2015). Perfiles de orientaciones de metas y autoconcepto de estudiantes de Educación Secundaria. Revista de Psicodidáctica, 20, 99-116. https://doi.org/10.1387/RevPsicodidact.1023

Kenny, M. E. y Hage, S. M. (2009). The next frontier: Prevention as an instrument of social justice. The Journal of Primary Prevention, 30(1), 1-10. https://doi.org/10.1007/s10935-008-0163-7

Lázaro, S.y López,F. (2010). Continuidad de los efectos del maltrato durante la infancia en adolescentes acogidos en centros de protección. Infancia y Aprendizaje, 33, 255-268. https://doi.org/10.1174/021037010791114599

Lippman, L. H., Anderson Moore, K., Guzman, L., Ryberg, R., McIntosh, H., Ramos, M. F., ... Kuhfeld, M. (2014). Flourishing children: Defining and testing indicators of positive development. Nueva York, Estados Unidos: Springer Science.

Liu, Y., Wang, Z. y Lü, W. (2013). Resilience and affect balance as mediators between traitemotional intelligence and life satisfaction. Personality and Individual Differences, 54, 850-855. https://doi.org/10.1016/j. paid.2012.12.010

Mérida, R., Serrano, A. y Tabernero, C. (2015). Diseño y validación de un cuestionario para la evaluación de la autoestima en la infancia. Revista de Investigación Educativa, 33, 149-162.

Mora M. y Raich, R. M. (2014).Autoestima: evaluación y tratamiento. Madrid, España: Editorial Síntesis.

Morton, M. H. y Montgomery, P. (2013). Youth empowerment programs for improving adolescents' self-efficacy and self-esteem: A systematic review. Research on Social Work Practice, 23, 22-33.

Mruk, C. J. (2006). Self-esteem research, theory and practice: Toward a positive psychology of self-esteem. Nueva York, Estados Unidos: Springer.

O’Mara, A. J., Marsh, H. W., Craven, R. G. y Debus, R. L. (2006). Do self-concept interventions make a difference? A synergistic blend of construct validation and meta-analysis. Educational Psychologist, 41, 181-206. https://doi.org/10.1207/s15326985ep4103_4

Prince-Embury, S. y Saklofske, D. H. (2014). Resilience interventions for youth in diverse populations. Nueva York, NY, Estados Unidos: Springer Science+Business Media. https://doi.org/10.1007/978-1-4939-0542-3

Ramos-Díaz, E., Rodríguez-Fernández, A., Fernández-Zabala, A., Revuelta, L., y Zuazagoitia, A. (2016). Apoyo social percibido, autoconcepto e implicación escolar de estudiantes adolescentes. Revista de Psicodidáctica, 21, 339-356. https://doi.org/10.1387/RevPsicodidact.14848

Rodríguez-Fernández, A., Ramos-Díaz, E., Fernández-Zabala, A., Goñi, E., Esnaola, I. y Goñi, A. (2016). Contextual and psychological variables in a descriptive model of subjective well-being and school engagement. International Journal of Clinical and Health Psychology, 16(2), 166-174. https://doi.org/10.1016/j. ijchp.2016.01.003

Shaffer, A., Huston, L. y Egeland, B. (2008). Identification of child maltreatment using prospective and self-report methodologies: A comparison of maltreatment incidence and relation to later psychopathology. Child abuse and Neglect, 32, 682-692. https://doi.org/10.1016/j.chiabu.2007.09.010

Shavelson, R. J., Hubner, J. J. y Stanton, G. C. (1976). Self-concept: Validation of construct interpretations. Review of Educational Research, 46, 407-441. https://doi.org/10.2307/1170010

Wright, M., Masten, A. S. y Narayan, A. J. (2013). Resilience processes in development: Four waves of research on positive adaptation in the context of adversity. En S. Goldstein y R. B. Brooks (Eds.), Handbook of resilience in children (pp. 15-37). Nueva York, Estados Unidos: Springer Science.

Artículo recibido: 16/07/2017

Revisión recibida: 14/11/2017

Artículo aceptado: 17/01/2018 